\title{
DISCUSSION
}

\section{Are all new drugs 'healthy'?}

SUJITH J CHANDY*, ATIYA R FARUQUI**

*Reader and ${ }^{* *}$ MD student, Department of Pharmacology and Clinical Pharmacology, Christian Medical College, Vellore 632004, India.

e-mail: clinpharm@cmcvellore.ac.in

The race for the launch of new drugs, brands and combination drugs has resulted in nearly 50-80 new entries in the market in the year 2002 alone. Is it 'healthy' to have such a flooding of the market with new drugs? Do these new drugs really have benefits over their older congeners as most of them claim to have? To answer these questions, we need to look at two important aspects in the field of new drug marketing: safety and claims of superiority.

\section{Safety of new drugs}

The health sector is faced with the issue of safety of all new drugs. In the present system, before a new drug is introduced into the market, it is mandatory to go through different stages of laboratory and clinical trials. These are aimed at establishment of therapeutic efficacy as well as the safety profile of the new drug. The various phases of the clinical trial are (1):

Phase I trials test the drug on a sample of 25-50 healthy volunteers to establish its safety. Phase II trials test 50300 patients for the drug's therapeutic effect. In Phase III trials, between 250 and 1,000 patients participate in a randomized clinical trial comparing the drug with a placebo to confirm the drug's efficacy. Phase IV 'trials' are post-marketing surveillance of the drug's safety in the general population and can cover between 2,000 and 10,000 people.

From these figures, one can see that the number of people actually exposed to the drug in the first three phases are few (a maximum of 1,350). If there are no serious adverse effects at this point, the drug is approved for marketing.

Is a number of 1,350 enough to detect a rare adverse effect? Let us examine this issue by looking at the following situation: In a case where the adverse drug reaction to a particular drug has an incidence of 1 in 1,000 (occurs in 1 patient out of 1,000 treated), we would need to screen a minimum number of 3,600 patients for the confirmation of its absence (2).

As 3,600 people are usually not involved in the first three phases of a clinical trial, it is possible that a drug with a rare but fatal adverse reaction could be used by the general population. The process of monitoring for safety should thus continue. This monitoring is done in Phase IV, termed as 'post-marketing surveillance'. The spectrum of adverse effects of any drug range from the common (detected during these trials and documented), to the rare (unidentified in early studies and yet important). It is these rare side-effects, usually undocumented in trial settings, which may subsequently have serious consequences for the population taking that drug. This makes the postmarketing surveillance an even more important tool to get the entire spectrum of the drug's activity.

It takes some time before such large numbers of patients get treated and observed. Thus, we see a delay of many years before such significant reactions are reported. The risk of 1 in 1,000 went undetected for 3-7 years in the following instances: pulmonary embolism due to oral contraceptives, halothane-induced jaundice, lincomycininduced colitis (2).

In many occasions, the incidence of a potentially fatal adverse reaction may be smaller than this. This therefore means that it may be even more difficult to detect them in the population and would need a larger number of patients on the drug, and a more intensive surveillance programme.

There are a few other issues to be highlighted regarding post-marketing surveillance. The first issue, that of who conducts the surveillance and who are the subjects, is an important one with respect to its credibility. In most of the cases, these are conducted by pharmaceutical companies, and therefore the issue of potential bias comes into play. The other aspect is that the surveillance done in western countries for adverse effects is often extrapolated to the Indian population. Considering the genetic differences as well as diet and nutrition, the extrapolation of such reports may not give the true picture in the Indian setting.

\section{Claims of superiority}

Post-marketing surveillance is not only about reporting adverse effects. It is also done to see the performance of these drugs as compared to the existing ones. Often, 
doctors are exposed to various claims of pharmaceutical companies that their drug is 'superior' to existing drugs, with the standard phrase being 'the initial results are very promising'. Hence, it is essential to consider the factors affecting the feedback from the case reports of patients receiving any new drug. One of the most important factors emerges when post-marketing surveillance is subject to an effect called the Weber Effect (3). This phenomenon, first described by Dr Peter Weber, denotes the combined effects of rapid increase in the use of, and interest in, a new drug, leading to a high rate of reporting. However, comparisons with older drugs with stable reporting can be misleading. Therefore, both the general over-reporting, for the new drug, combined with the Weber Effect, should be considered when comparing new drugs with drugs already existing in the market.

\section{A possible solution}

In the Indian scenario, general practitioners, with an outpatient base and limited facilities for adverse drug reaction (ADR) monitoring tend to be among the first ones to use these 'latest' drugs. Even the patients seem to prefer these 'new' drugs for their treatment without realising the risk that they are putting themselves through.

The solution to most of the above problems associated with the use of new drugs can be derived from the example of the policies of the Japanese health ministry (4). In their set-up, the pharmaceutical companies are required to provide their product to a limited number of medical institutions for a period of three months following the launch of a product and conduct focused post-marketing surveillance. After inspection of the results of this surveillance, the companies are then allowed to expand the number of medical institutions using the product. Requiring companies to limit sales to a small number of medical institutions and collecting information in this way makes it easier to respond to adverse drug reactions of newly approved products. It needs to be examined whether this practice can be transcribed to the Indian situation.

It is imperative that the drug control authorities and other governmental agencies strengthen the post-marketing surveillance programme in India. They need to look at ways of minimising risk to the population due to rare adverse events when new drugs are introduced into the market. Any doctor or hospital, when deciding on the use of a new drug, should objectively compare it to the existing drugs. A balanced judgement needs to be made without blindly following the tall claims of superiority made by drug manufacturers.

In conclusion, an awareness of the importance of postmarketing surveillance is the need of the hour, at all levels, whether it be the government, the health professional or the patient. An official surveillance programme needs to be initiated and subsequently evaluated. Till then the question remains, 'Are all new drugs 'healthy'?'

\section{References}

1. Laurence DR and Bennet PN. Clinical Pharmacology. 7th ed. Edinburg,UK: Churchill Livingstone, 1997:42.

2. Ferner RE, Hazards, risks and reality. Br J Clin Pharmacol 1992;33:125-8.

3. WHO international drug monitoring: cerivastatin and gemfibrozil. WHO Drug Information 2002;16:8-11.

4. Postmarketing system to be revised. WHO Drug Information 1999;13:240.

\section{The Seventh World Congress of Bioethics}

The Seventh World Congress of Bioethics of the International Association of Bioethics will be held at the University of New South Wales in Sydney, Australia from November 9 to 12, 2004. The theme of the Congress is Deep Listening: bridging divides in local and global ethics. The sub-themes are: indigenous health ethics, public health ethics from local and global perspectives, and refiguring the body.

The theme Deep Listening was suggested by dadirri-a word from the Australian Aboriginal language of the Ngangikurungkurr tribe-and suggests a conversation of discovery between cultures and between those with power and the disempowered.

Invitations are extended to present a paper, special symposium, conversation piece, or poster in any of the wide range of areas covered by the Congress.

The University of New South Wales will also host satellite conferences including the International Network on Feminist Approaches to Bioethics 2004 and the 10th Conference of the Australasian Bioethics Association (ABA).

For more details, please visit the official Congress website: www.bioethicsworldcongress.com. 